\title{
Backscatter in stratified turbulence
}

\author{
Sina Khani ${ }^{1}$ \\ Wind Engineering and Renewable Energy Laboratory (WiRE), École Polytechnique Fédérale \\ de Lausanne (EPFL), Lausanne, Switzerland CH-1015 \\ Department of Applied Mathematics, University of Waterloo, Waterloo, Ontario, Canada \\ N2L $3 G 1$ \\ Michael L. Waite \\ Department of Applied Mathematics, University of Waterloo, Waterloo, Ontario, Canada \\ N2L $3 G 1$
}

\begin{abstract}
In this paper, kinetic and potential energy transfers around a spectral test filter scale in direct numerical simulations of decaying stratified turbulence are studied in both physical and spectral domains. It is shown that while the domain-averaged effective subgrid scale energy transfer in physical space is a net downscale cascade, it is actually a combination of large values of downscale and upscale transfer, i.e. forward- and backscatter, in which the forward scatter is slightly dominant. Our results suggest that spectral backscatter in stratified turbulence depends on the buoyancy Reynolds number $R e_{b}$ and the filtering scale $\Delta_{\text {test }}$. When the test filter scale $\Delta_{\text {test }}$ is around the dissipation scale $L_{d}$, transfer spectra show spectral backscatter from sub-filter to intermediate scales, as reported elsewhere. However, we find that this spectral backscatter is due to viscous effects at vertical scales around the test filter. It is also shown that there is a non-local energy transfer from scales larger than the buoyancy scale $L_{b}$ to small scales. The effective turbulent Prandtl number spectra demonstrate that the assumption $P r_{t} \approx 1$ is reasonable for the local energy transfer.
\end{abstract}

Keywords: Stratified turbulence; Direct numerical simulations;

up- and downscale energy transfer

${ }^{1}$ email: sinakhani@uwaterloo.ca

Preprint submitted to European Journal of Mechanics B/Fluids

July 1, 2016

(c) 2016. This manuscript version is made available under the CC-BY-NC-ND 4.0 license

http://creativecommons.org/licenses/by-nc-nd/4.0/ 


\section{Introduction}

Large eddy simulations (LES) is an approach for decreasing the computational costs of direct numerical simulations (DNS) of turbulent flows. In LES, the large energy-containing eddies are directly resolved but subgrid scale (SGS)

5 eddies are parametrized. Most SGS parameterizations such as the Smagorinsky 1] and Kraichnan 2] models are based on the Richardson [3] energy-cascade hypothesis, which argued that turbulent kinetic energy is generated at large scales and dissipated at small scales. Richardson's prediction may be valid for the average kinetic energy cascade, but might not be accurate in the local sense. Locally, the energy cascade is the net outcome of forward scatter, i.e. the energy transfer from large to small scales, and the backscatter, which is the reverse energy transfer from small to large scales. For example, Piomelli et al. [4] and Domaradzki et al. [5] have shown that forward- and backscatter are of the same order of magnitude in turbulent channel flow and isotropic decaying turbulence, respectively.

The dynamic SGS scheme, proposed by Germano et al. [6], has been designed to improve the performance of purely dissipative eddy viscosity SGS schemes such as the Smagorinsky [1] model by considering a time- and spacedependent dynamic Smagorinsky coefficient $c_{s}$ with negative values corresponding to backscatter. However, averaging $c_{s}$ over a homogeneous direction is often required to avoid numerical instabilities [e.g. 7, 8, 9]. Information about the dynamics of the local energy transfer is therefore lost, and in practice, the averaging procedure removes the local effect of backscatter, because the averaged $c_{s}$ is usually positive [e.g. 7, 9, 10, 11, 12].

Stratified turbulence is a model for turbulence in the atmospheric mesoscale and oceanic sub-mesoscale, at which fluid motions are strongly affected by stratification but weakly affected by the Earth's rotation [e.g. 13]. The presence of stratification leads to the generation of anisotropic features such as pancake vortices, which in turn lead to the development of different length scales and spec- 
tral slopes in the horizontal and vertical directions [e.g. 14, 15, 16, 17, 18, 19].

Recently, the dynamics of energy transfer between large and sub-filter scales in stratified turbulence has been studied in wavenumber space [20, 21]. Using DNS of decaying stratified turbulence, Khani \& Waite [20] studied the dynamics of horizontal and vertical energy transfer around the Ozmidov scale. It was shown

35 that stratification leads to a non-local energy transfer from large to small horizontal scales when an anisotropic horizontal test cutoff $k_{c}$ is employed [20]. In addition, the spectral eddy viscosity based on the vertical kinetic energy shows negative values when the flow is subjected to very stable stratification, leading to negative effective turbulent Prandtl number [21].

The performance of different eddy viscosity SGS models (the Smagorinsky, dynamic Smagorinsky and Kraichnan models) has recently been studied in LES of stratified turbulence with isotropic grid resolution [10, 22]. In all cases, it is shown that LES must resolve the buoyancy scale $L_{b}=2 \pi u_{r m s} / N$ to capture the fundamental large scale characteristics of stratified turbulence. Here, $u_{r m s}$ and ${ }_{45} \quad \mathrm{~N}$ are the root-mean-square velocity and buoyancy frequency. The resolution criterion for LES of stratified turbulence depends on the SGS model: it requires $\Delta<0.47 L_{b}$ for the Kraichnan model, $\Delta<0.24 L_{b}$ for the dynamic Smagorinsky model and $\Delta<0.17 L_{b}$ for the regular Smagorinsky model [where $\Delta$ is the grid spacing, 10, 22]. These criteria are obtained by studying the capability of differ-

so ent SGS models to capture three fundamental features of stratified turbulence: layered structures that break down into Kelvin-Helmholtz (KH) instabilities and small-scale turbulence when shear is large; horizontal wavenumber energy spectra with an approximately $-5 / 3$ power law at large scales along with a bump (or shallowness) around $k_{b}$; and the resolution of regions with small and negative Richardson number, which demonstrate the presence of $\mathrm{KH}$ instabilities, overturning and small-scale turbulence. These fundamental features have been reported in several DNS and hyperviscosity simulations of stratified turbulence [e.g. 14, 15, 17, 18, 19, 20, 23, 24, 25, 26]. The importance of $L_{b}$ implies that LES of stratified turbulence does not require resolution of the smaller Ozmidov ${ }_{60}$ scale $L_{o}=2 \pi\left(\epsilon / N^{3}\right)^{1 / 2}$, where $\epsilon$ is the kinetic energy dissipation rate [10, 22]. 
As a result, the potential of employing LES with much coarser grids than DNS is promising.

In physical space, backscatter can be calculated from DNS data by filtering velocity fields, and directly measuring the sub-filter scale (SGS) momentum tensor $\tau_{i j}$. Following Piomelli et al. [4], backscatter may be defined by negative values of the effective SGS dissipation rates $\epsilon_{S G S}$ and $\varepsilon_{S G S}$, written as

$$
\epsilon_{S G S}=-2 \tau_{i j} \bar{S}_{i j}, \quad \varepsilon_{S G S}=-h_{j} \frac{\partial \bar{\rho}}{\partial x_{j}}
$$

where $\bar{S}_{i j}$ is the filtered rate of strain. A similar procedure was used recently to analyze SGS backscatter in DNS of reacting turbulence [27], which found that backscatter depends on the dynamics of reacting flows and hence is not just a random and intermittent process. The dynamics of backscatter has not been studied for stratified turbulence. Indeed, the physical mechanisms underlying the dynamics of energy transfer are not completely understood in this context.

In this paper, the dynamics of forward and inverse energy transfer around the Ozmidov scale in DNS of stratified turbulence is studied at different buoyancy netic and potential energy transfer, and the effective turbulent Prandtl number, are analyzed. The governing equations of motion and formulations for analyzing DNS diagnoses are presented in $\$ 2$. Section 3 presents the methodology used, and $\$$ presents the results and discussion. Conclusions are given in $\$ 5$,

\section{Governing equations}

The non-dimensional Boussinesq equations are

$$
\begin{array}{r}
\frac{\partial \boldsymbol{u}}{\partial t}+\boldsymbol{u} \cdot \nabla \boldsymbol{u}=-\nabla p-\frac{1}{F r_{\ell}{ }^{2}} \rho \boldsymbol{e}_{z}+\frac{1}{R e_{\ell}} \nabla^{2} \boldsymbol{u}, \\
\nabla \cdot \boldsymbol{u}=0, \\
\frac{\partial \rho}{\partial t}+\boldsymbol{u} \cdot \nabla \rho-w=\frac{1}{\operatorname{Re}_{\ell} \operatorname{Pr}} \nabla^{2} \rho,
\end{array}
$$

where $\boldsymbol{u}=(u, v, w)$ is the velocity vector, $\rho$ and $p$ are the density and pressure perturbations, respectively; $R e_{\ell}, F r_{\ell}$, and $\operatorname{Pr}$ are the initial Reynolds, Froude 
and Prandtl numbers, respectively, which are defined in terms of the initial velocity and length scales. The density perturbation is nondimensionalized in terms of the constant background density gradient and the initial length scale. We can define the test-filtered variables by applying a filtering operator to the DNS results, e.g. for velocity

$$
\overline{\boldsymbol{u}}(\boldsymbol{x}, t)=\int_{D} G(\dot{\boldsymbol{x}}, \boldsymbol{x}) \boldsymbol{u}(\dot{\boldsymbol{x}}, t) \mathrm{d} \dot{\boldsymbol{x}},
$$

where $G$ is the filtering function and $D$ is the spatial domain. Hence, the governing equations (24) can be rewritten for the test-filtered variables as follows

$$
\begin{array}{r}
\frac{\partial \bar{u}_{i}}{\partial t}+\frac{\partial}{\partial x_{j}}\left(\bar{u}_{i} \bar{u}_{j}\right)=-\frac{\partial \bar{p}}{\partial x_{j}}-\frac{1}{F r_{\ell}{ }^{2}} \bar{\rho} e_{z}+\frac{1}{R e_{\ell}} \frac{\partial^{2} \bar{u}_{i}}{\partial x_{j} \partial x_{j}}-\frac{\partial \tau_{i j}}{\partial x_{j}}, \\
\frac{\partial \bar{u}_{i}}{\partial x_{i}}=0 \\
\frac{\partial \bar{\rho}}{\partial t}+\frac{\partial}{\partial x_{j}}\left(\bar{\rho} \bar{u}_{j}\right)-\bar{w}=\frac{1}{R e_{\ell} \operatorname{Pr}} \frac{\partial \bar{\rho}}{\partial x_{j} \partial x_{j}}-\frac{\partial h_{j}}{\partial x_{j}}
\end{array}
$$

where

$$
\tau_{i j}=\overline{u_{i} u_{j}}-\bar{u}_{i} \bar{u}_{j}, \quad h_{j}=\overline{\rho u_{j}}-\bar{\rho} \bar{u}_{j},
$$

are the SGS momentum and density fluxes which are known since DNS resolves both the test-filtered and sub-test-filter scales. Using the measured SGS momentum flux $\tau_{i j}$, we can calculate the SGS dissipation field $\epsilon_{S G S}$ through equation (10), which gives the local rate of energy transfer between the test-filtered scales and the sub-test-filter motions. Following Piomelli et al. [4], if $\epsilon_{S G S}$ is positive, then the kinetic energy transfers from the test-resolved to the SGS motions (forward scatter); however, if $\epsilon_{S G S}$ is negative, kinetic energy is transferred in the opposite direction (backscatter), i.e. $\epsilon_{S G S}=\epsilon^{+}+\epsilon^{-}$, where

$$
\epsilon^{ \pm}=\frac{1}{2}\left(\epsilon_{S G S} \pm\left|\epsilon_{S G S}\right|\right)
$$

Similarly, forward- and backscatter of potential energy is

$$
\varepsilon^{ \pm}=\frac{1}{2}\left(\varepsilon_{S G S} \pm\left|\varepsilon_{S G S}\right|\right) .
$$

In the wavenumber domain, assuming periodic boundary conditions and a sharp spectral filter with wavenumber $k_{c}$, we can use the measured SGS fluxes in 
(9) to calculate the kinetic and potential effective SGS energy transfer following Pope [9] as

$$
\begin{array}{r}
T_{k}\left(k \mid k_{c}, t\right)=\frac{1}{2}\left\langle F_{j} \hat{\bar{u}}_{j}^{*}+F_{j}^{*} \hat{\bar{u}}_{j}\right\rangle_{k}, \\
T_{p}\left(k \mid k_{c}, t\right)=\frac{1}{2}\left\langle J \hat{\bar{\rho}}^{*}+J^{*} \hat{\bar{\rho}}\right\rangle_{k},
\end{array}
$$

where $F_{j}$ and $J$ are the Fourier coefficients of the SGS fluxes $\partial \tau_{i j} / \partial x_{j}$ and $\partial h_{j} / \partial x_{j}$, respectively, where $*$ is complex conjugate and the angle bracket $\langle\cdots\rangle_{k}$ denotes summing of the Fourier modes over spherical shells of constant radius $k$. Here, $k=|\boldsymbol{k}|$ and $\boldsymbol{k}=\left(k_{x}, k_{y}, k_{z}\right)$ is the three dimensional wavevector. The $\mid k_{c}$ notation in (12,13) underlines that the transfers are computed relative to a particular filter wavenumber [9]. The physical and spectral SGS energy transfer

95 are related: the sum of the SGS transfer spectra equals the domain-averaged SGS dissipation rate, written as

$$
\begin{aligned}
& \sum_{k} T_{k}\left(k \mid k_{c}, t\right)=\epsilon_{S G S}\left(k_{c}, t\right), \\
& \sum_{k} T_{p}\left(k \mid k_{c}, t\right)=\varepsilon_{S G S}\left(k_{c}, t\right),
\end{aligned}
$$

where the sum is over all wavenumbers resolved by the filter. However, there is not a straightforward connection between the spectral and physical backscatter (i.e. negative values of $T_{k}, T_{p}, \epsilon_{S G S}$ and $\varepsilon_{S G S}$ ) since they result from different, physical vs. wavenumber, perspectives. Nevertheless, the wavenumbers associated with negative transfers, i.e. spectral backscatter, likely give some indication of the length scales associated with $\epsilon^{-}$.

In addition, the effective eddy viscosity $\nu_{e}\left(k \mid k_{c}, t\right)$ and the effective eddy diffusivity $D_{e}\left(k \mid k_{c}, t\right)$ are defined as

$$
\nu_{e}\left(k \mid k_{c}, t\right)=\frac{T_{k}\left(k \mid k_{c}, t\right)}{2 k^{2} E(k, t)}, \quad D_{e}\left(k \mid k_{c}, t\right)=\frac{T_{p}\left(k \mid k_{c}, t\right)}{2 k^{2} E_{p}(k, t)},
$$

where $E(k, t)$ and $E_{p}(k, t)$ are the kinetic and potential energy spectra. Using these, the effective turbulent Prandtl number can be written as

$$
\operatorname{Pr}_{t}\left(k \mid k_{c}, t\right)=\frac{\nu_{e}\left(k \mid k_{c}, t\right)}{D_{e}\left(k \mid k_{c}, t\right)}
$$


Stratified turbulence is characterised by large Reynolds number $R e=u_{r m s} l_{h} / \nu$ and small horizontal Froude number $F r_{h}=u_{r m s} / N l_{h}$ such that the buoyancy Reynolds number $\operatorname{Re}_{b}=\operatorname{ReFr}_{h}^{2}$ is also large [e.g. 17]. Here, $l_{h}$ and $\nu$ are the horizontal length scale and molecular viscosity, respectively. The dissipation length scale is given by $k_{d}=\left(\epsilon / \nu^{3}\right)^{1 / 4}$. For decaying turbulence, the maximum ${ }_{110} k_{d}$ and $R_{b}$ happen at the time when $\epsilon$ is maximum.

\section{Methodology}

An idealized case study for decaying stratified turbulence is designed in a cubic domain with triply periodic boundary conditions with side length $L=2 \pi$. Taylor-Green (TG) vortices of size $\pi$ are used as initial conditions, i.e.

$$
\boldsymbol{u}(\boldsymbol{x}, 0)=\cos (z)[\cos (x) \sin (y),-\sin (x) \cos (y), 0] .
$$

Also, approximately $10 \%$ of the initial energy in the form of low-level noise perturbs the initial TG vortices isotropically. Noise is restricted to small wavenumbers $k<10$. The domain is smaller than that used in Khani \& Waite 20] to allow for higher effective spatial resolution, which leads to a decrease in computation time [as in e.g. 5]. The spectral transform method is employed for computing spatial derivatives, along with the two-thirds rule to eliminate aliasing errors from $x, y$ and $z$ directions. The number of grid points is given by $n$, and so the effective grid spacing is $\Delta=1.5 \mathrm{~L} / \mathrm{n}$ and the maximum wavenumber is $k_{\max }=n / 3$ in both horizontal and vertical directions. Resolutions are chosen to resolve the Kolmogorov dissipation wavenumber $k_{d}$, with $k_{\max } / k_{d}>0.7$ in all cases. The explicit third-order Adams-Bashforth scheme is used for timestepping of all terms except the diffusion terms, for which the implicit trapezoidal method is employed.

125 Simulations with six different initial Reynolds number $R e_{\ell}=1350$, 3030, 6400, 10900, 14700 and 18000, and a range of the initial Froude number $F r_{\ell}$ from 0.12 to $\infty$ are considered. These parameters yield layer thickness, which are given by the buoyancy scale [e.g. 19, 20], that are several times smaller than 
the domain height (i.e. buoyancy wavenumber $k_{b}=2 \pi / L_{b}>1$ ). Four different sharp spectral test cutoff filters $k_{c}=20,40,80$ and 160 are employed to study the spectral energy transfer between scale. The horizontal length scale $l_{h}$ is calculated using the Taylor hypothesis, $u_{r m s}$ is set to $\sqrt{E(t)}$ because the vertical kinetic energy is relatively small, and $\operatorname{Pr}=1$. The simulations proceed similarly to other studies of TG stratified turbulence [e.g. 20, 23, 25]: the initial vortices evolve into thin pancake vortices which ultimately, for $R e_{b} \gtrsim 1$, develop $\mathrm{KH}$ instabilities and patches of small-scale turbulence. Table 1 gives the parameters and identifiers for each simulation, including $R e_{\ell}, F r_{\ell}$ and $n$; range of turbulent buoyancy Reynolds and horizontal Froude numbers from when $\epsilon$ is maximum up to the end of simulation; ratio $k_{\max } / k_{d}$ where $k_{d}=2 \pi / L_{d}$; buoyancy and Ozmidov wavenumbers $k_{b}$ and $k_{o}$, where $k_{o}=2 \pi / L_{o}$; the time interval $\Delta t_{\text {avg }}$ over which energy and transfer spectra are averaged; and the time at which the total viscous dissipation rate $\epsilon_{t o t}$ is maximum.

\section{Results \& discussion}

\subsection{Overview of simulations}

Figure 1shows time series of the total energy and dissipation rates, in the top and bottom panels, respectively, where the left panels correspond to cases with $R e_{b} \gtrsim 1$ and the right panels show those with $R e_{b} \lesssim 1$. For the unstratified case, a quick drop in the total energy level and a maximum in the total dissipation rate are visible at $t \approx 9$. Increased stratification or decreased Reynolds number postpones the time of the energy decrease and maximum dissipation. For example, the case with $R e_{\ell}=10900$ and $F r_{\ell}=0.16$ has only a small decrease in energy up to $t \approx 20$, while the maximum dissipation rate occurs around $t=22$ (figures $1 \mathrm{1}, \mathrm{c}$ ). These trends suggest that by increasing stratification or decreas155 ing the Reynolds number, i.e. by decreasing the buoyancy Reynolds number, the occurrence of turbulence is postponed [in line with 20, 23]. Interestingly, the 
Table 1: List of numerical simulations and parameters

\begin{tabular}{|c|c|c|c|c|c|c|c|c|c|c|}
\hline Identifier & $R e_{\ell}$ & $F r_{\ell}$ & $n$ & $R e_{b}$ & $F r_{h}$ & $k_{\max } / k_{d}$ & $k_{b}$ & $k_{o}$ & $\Delta t_{\text {avg }}$ & $t$ of $\epsilon_{t o t}^{\max }$ \\
\hline R147F0.6 & 14700 & 0.64 & 960 & $8.60-21.30$ & $0.024-0.033$ & $1.0-1.2$ & $5.1-9.4$ & $33.0-52.1$ & $10 \leq t \leq 14$ & 11.5 \\
\hline R180F0.5 & 18000 & 0.48 & 960 & $7.10-14.34$ & $0.023-0.027$ & $0.8-1.0$ & $7.8-12.0$ & $51.3-73.1$ & $16 \leq t \leq 20$ & 18.5 \\
\hline R109F0.1 & 10900 & 0.16 & 768 & $0.81-1.45$ & 0.009 & $0.9-1.0$ & $20.8-28.1$ & $217.4-290.6$ & $20 \leq t \leq 24$ & 21.5 \\
\hline R64F0.6 & 6400 & 0.64 & 384 & $4.15-10.13$ & $0.028-0.034$ & $0.7-0.9$ & $5.5-9.1$ & $31.5-49.2$ & $13 \leq t \leq 17$ & 14.1 \\
\hline R30F0.5 & 3030 & 0.48 & 512 & $1.30-2.47$ & $0.023-0.035$ & $1.7-2.0$ & $7.8-12.5$ & $50.7-69.8$ & $15 \leq t \leq 19$ & 17.6 \\
\hline R30F0.2 & 3030 & 0.24 & 512 & $0.33-1.00$ & $0.015-0.021$ & $1.5-2.0$ & $15.9-24.2$ & $113.0-197.5$ & $18 \leq t \leq 22$ & 20.2 \\
\hline R30F0.1 & 3030 & 0.12 & 512 & $0.12-0.19$ & 0.007 & $1.6-1.8$ & $29.5-37.6$ & $367.0-468.5$ & $25 \leq t \leq 29$ & 27.4 \\
\hline $\mathrm{R} 13 \mathrm{~F} 0.5$ & 1350 & 0.5 & 256 & $0.70-1.13$ & $0.033-0.041$ & $1.5-1.7$ & $9.0-12.8$ & $50.0-63.5$ & $20 \leq t \leq 24$ & 22.5 \\
\hline R30 & 3030 & $\infty$ & 512 & & & $1.2-2.1$ & & & $7 \leq t \leq 11$ & 8.8 \\
\hline
\end{tabular}



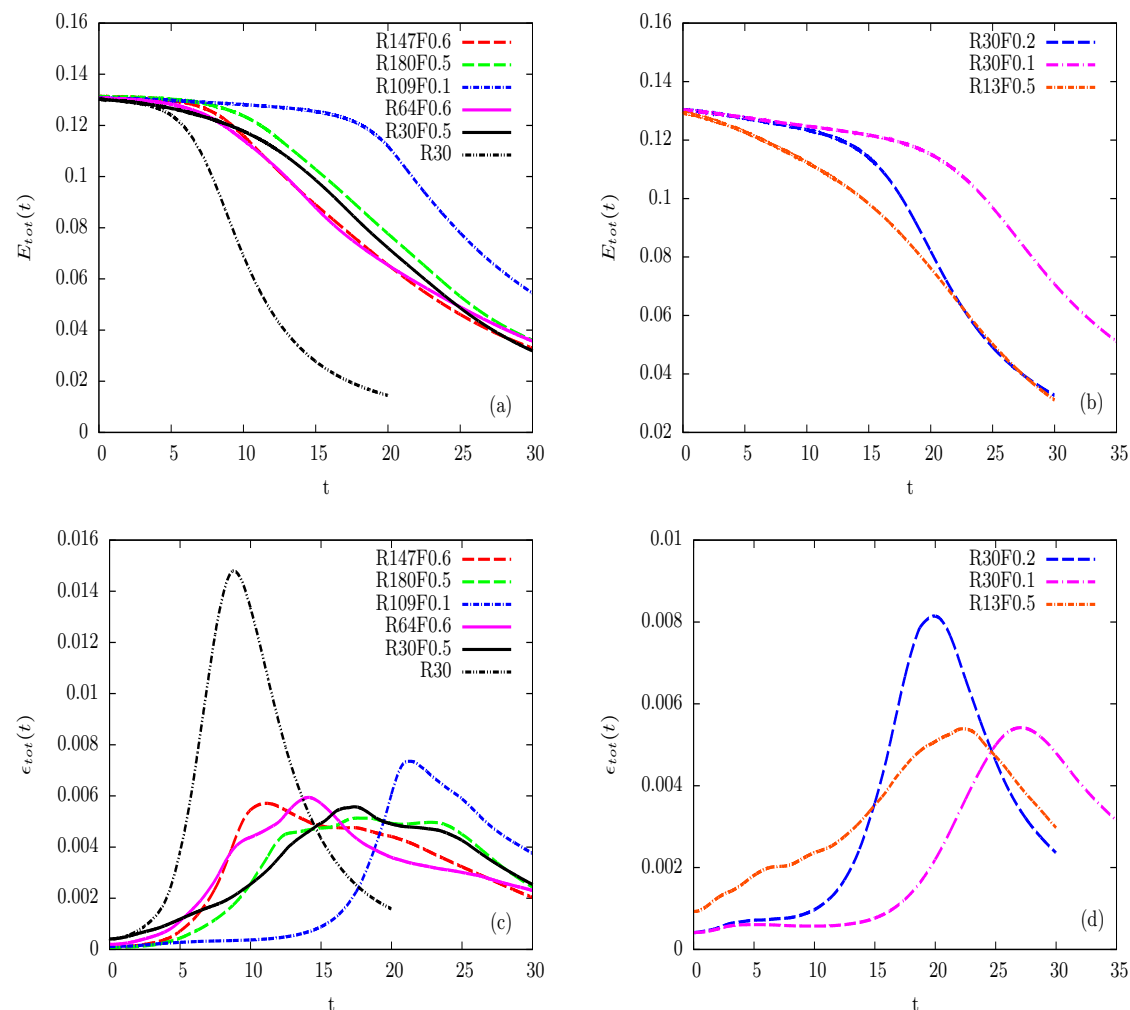

Figure 1: Time series of $(\mathrm{a}, \mathrm{b})$ total energy and $(\mathrm{c}, \mathrm{d})$ total dissipation rate. The left panels correspond to cases with $R e_{b} \gtrsim 1$ and the right panels show those with $R e_{b} \lesssim 1$ (see table 1 for the identifiers).

three cases with the highest $R e_{b}$ show very similar total energy and dissipation rate (i.e. solid green, solid magenta and dashed red lines in figures 1 1 , c). In addition, the simulation with $R e_{\ell}=3030$ and $F r_{\ell}=0.48$ has similar energy and dissipation time series to the case with the same initial Froude number and $R e_{\ell}=18000$ (solid black and dashed green curves in figures 1 $1 \mathrm{a}, \mathrm{c}$ ).

Figure 2(a,b) shows the normal horizontal component of the vorticity $\omega_{y}$ on the $x$ - $z$ plane for the high resolution case with $R e_{\ell}=14700$ and $F r_{\ell}=0.64$ at $t=15$, and the case with $R e_{\ell}=10900$ and $F r_{\ell}=0.16$ at $t=20$, which are around the time of maximum viscous dissipation. At strong stratification, $\omega_{y} \approx \partial u / \partial z$, so these figures show the $x$ component of the vertical shear. Lay- 
erwise structures along with $\mathrm{KH}$ instabilities are visible in both cases. In addition, small-scale features are present, which seem to correspond to the breakdown of KH billows into smaller-scale turbulence (the case with larger $R e_{b}$ has more regions with instabilities and turbulence as seen in figures 2 a,b). The time-averaged probability distribution of the local Richardson number $R i$ over $10 \leq t \leq 14$ for the high resolution case with $F r_{\ell}=0.64$, and over $20 \leq t \leq 24$ for the simulation with $R e_{\ell}=10900$ and $F r_{\ell}=0.16$ are shown in figure 2(c). The histograms are peaked around 0 and present a long tail for $R i>0$ and 175 a rapid drop off for negative $R i$. The points with small and negative local Richardson number suggest generation of KH instabilities and overturning, respectively (in line with figures $2 \mathrm{a}, \mathrm{b})$. It is shown that increased $R e_{b}$ leads to generation of more points with small and negative $R i$ (figure2). For smaller $R e_{b}$, regions of $\mathrm{KH}$ instabilities and overturning are reduced or suppressed entirely [not shown; see 30]. Similar behaviours are seen in LES of stratified turbulence in which increased resolution or decreased stratification leads to more resolved $\mathrm{KH}$ instabilities and overturning [10, 22].

The time-averaged kinetic energy and dissipation spectra are shown in figure 3 (similar to figure 1] left panels correspond to cases with $R e_{b} \gtrsim 1$ and right panels shows those with $R e_{b} \lesssim 1$ ). The time averaging is performed over a time interval that is approximately centered around the time of maximum $\epsilon_{t o t}(t)$ (as shown in table 1). Similar to previous studies [e.g. 20, 23, 28, 29], increased stratification or decreased Reynolds number steepens the slope of the spectra (figure 3). The spectral slopes for all simulations over the range $10 \leq k \leq 70$ are given in table 2. We use the least-squares method to measure these slopes. Overall, decreasing the buoyancy Reynolds number $R e_{b}$ suppresses the onset of turbulence and steepens the spectral power law (see table 2). Arrows in figure 3 indicate locations of the test cutoffs $k_{c}=20,40,80$ and 160 (the latter two only for the larger $R e_{b}$ cases), which are used in the next sections for measuring 

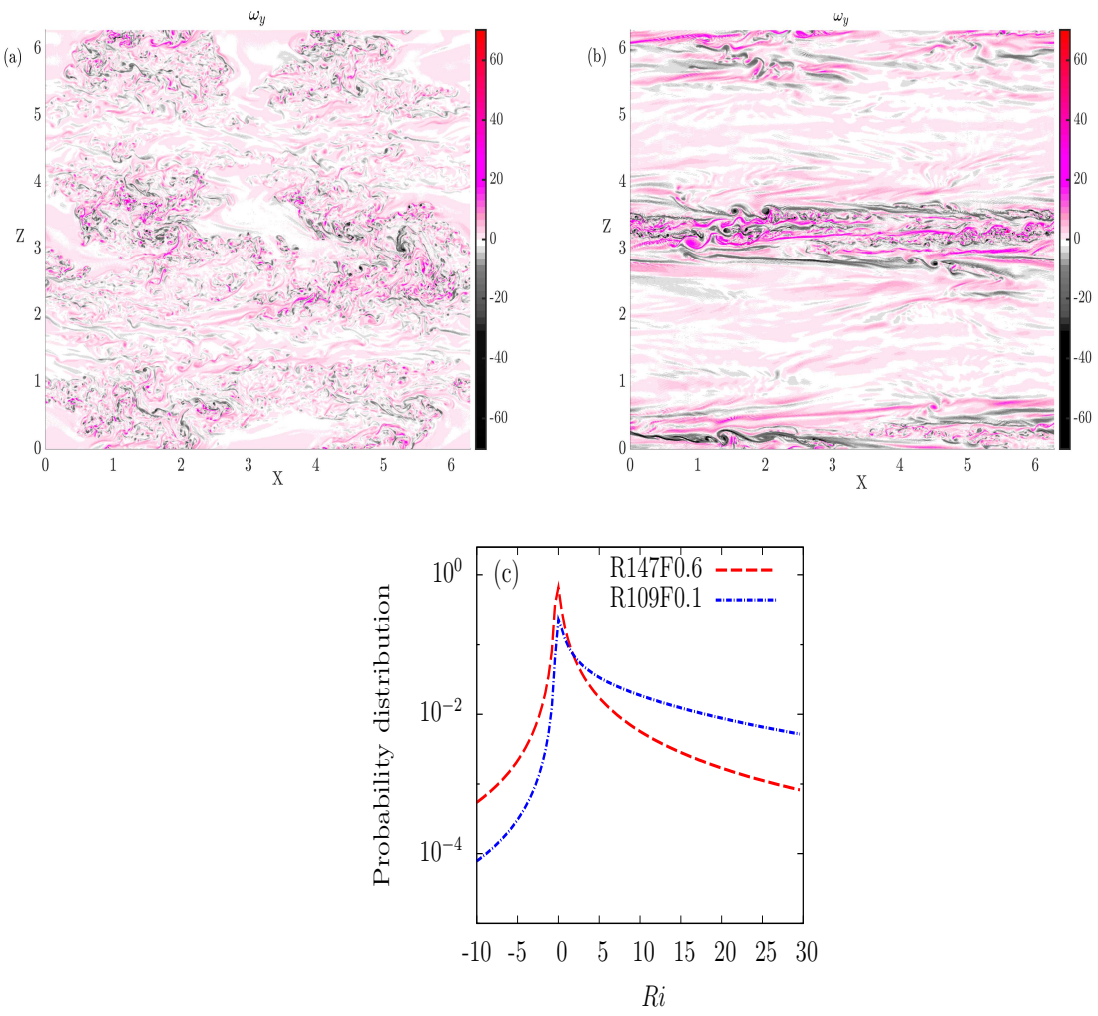

Figure 2: The horizontal component of vorticity field $\omega_{y}$ on the $x-z$ plane at $y \approx 0.25$ (a) for the high resolution case with $R e_{\ell}=14700$ and $F r_{\ell}=0.64$ at $t=15$, and (b) for the case with $R e_{\ell}=10900$ and $F r_{\ell}=0.16$ at $t=20$. (c) Averaged probability distribution of the local Richardson number $R i$ over $10 \leq t \leq 14$ for the case in (a) and over $20 \leq t \leq 24$ for the case in (b) - only the segment $-10 \leq R i \leq 30$ is shown. Histograms are normalized by bin size to get the probability distribution: 100 bins over $-10 \leq R i \leq 30(\Delta R i=0.4)$. 

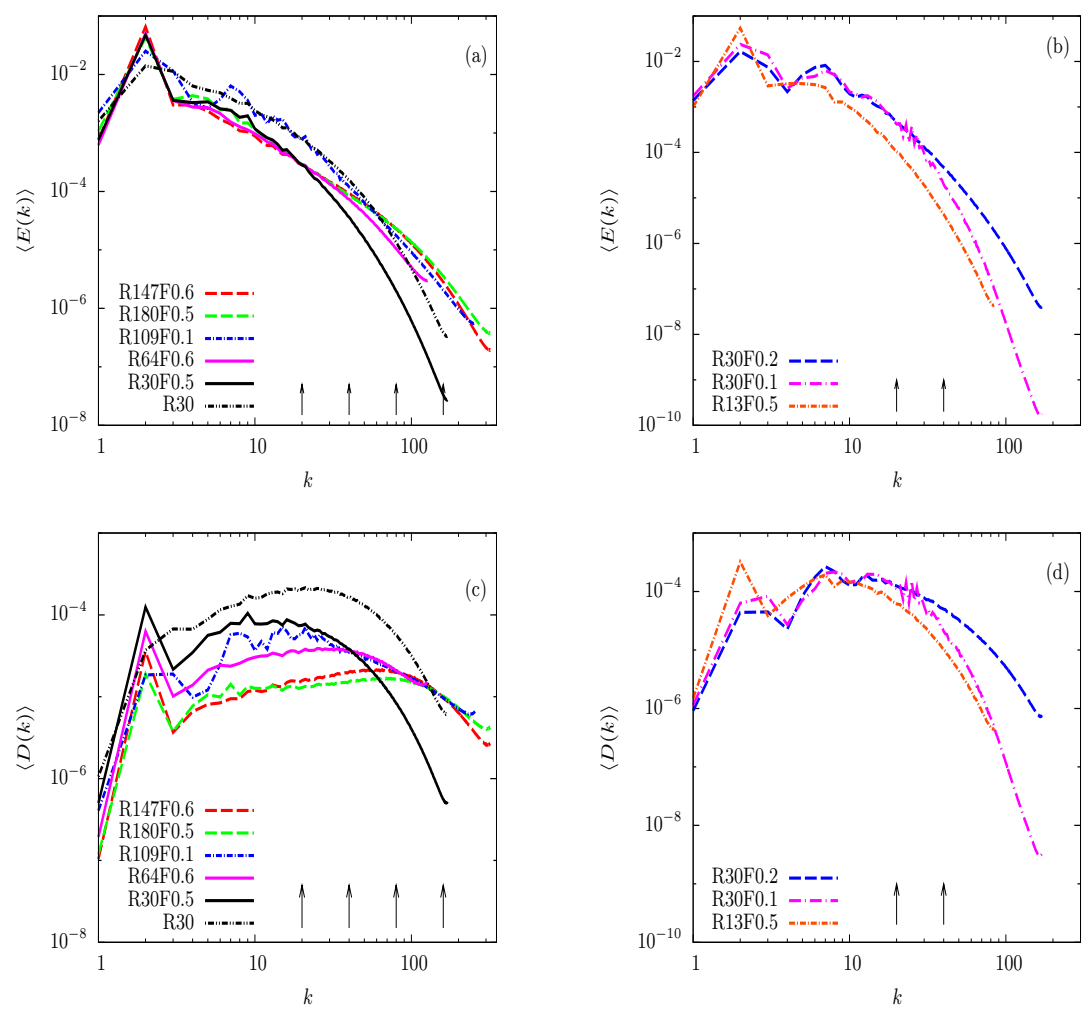

Figure 3: (a,b) Time-averaged kinetic energy spectra and (c,d) time-averaged dissipation spectra $D(k)=2 \nu k^{2} E(k)$. The left panels correspond to cases with $R e_{b} \gtrsim 1$ and right panels show those with $R e_{b} \lesssim 1$. Arrows indicate the location of the test cutoff $k_{c}=20,40,80$ and 160. 


\begin{tabular}{|c|c|}
\hline Identifier & Spectral slope \\
\hline R147F0.6 & -1.7 \\
\hline $\mathrm{R} 180 \mathrm{~F} 0.5$ & -1.8 \\
\hline R109F0.1 & -2.5 \\
\hline R64F0.6 & -2.1 \\
\hline R30F0.5 & -3.1 \\
\hline R30F0.2 & -3.3 \\
\hline R30F0.1 & -4.7 \\
\hline $\mathrm{R} 13 \mathrm{~F} 0.5$ & -4.8 \\
\hline R30 & -2.5 \\
\hline
\end{tabular}

\subsection{Energy transfer in physical space}

The forward- and backscatter components of the effective SGS kinetic and potential dissipation rates $\epsilon_{S G S}$ and $\varepsilon_{S G S}$ for $k_{c}=40$ are shown in figure 4 (left panels for cases with $R e_{b} \gtrsim 1$ and right panels for those with $R e_{b} \lesssim 1$ ). The maximum forward- and backscatter happens around the time when the total kinetic plus potential energy dissipation rate $\epsilon_{t o t}$ is peaked (see figures 15,d). Increased stratification or decreased Reynolds number decreases the amount of forward- and backscatter, and also postpones the peaks. As a result, decreased $R e_{b}$ reduces forward- and backscatter, and weakens their peak values (figure 4).

These results demonstrate that the presence of stratification decreases energy transfer between large and sub-filter scales, i.e. both up- and downscale energy transfers are reduced by increasing stratification [28, 29]. As seen above for the viscous dissipation rates, the three simulations with largest $R e_{b}$ have similar $\epsilon^{ \pm}$ and $\varepsilon^{ \pm}$time series, which peak at an earlier time than all the other stratified cases.

Figure 5 shows the net kinetic and potential effective SGS energy transfers (i.e. $\epsilon_{S G S}$ and $\left.\varepsilon_{S G S}\right)$ with $k_{c}=40$. Since the net transfer values are positive, this figure demonstrates that the effective SGS energy transfer is downscale, 

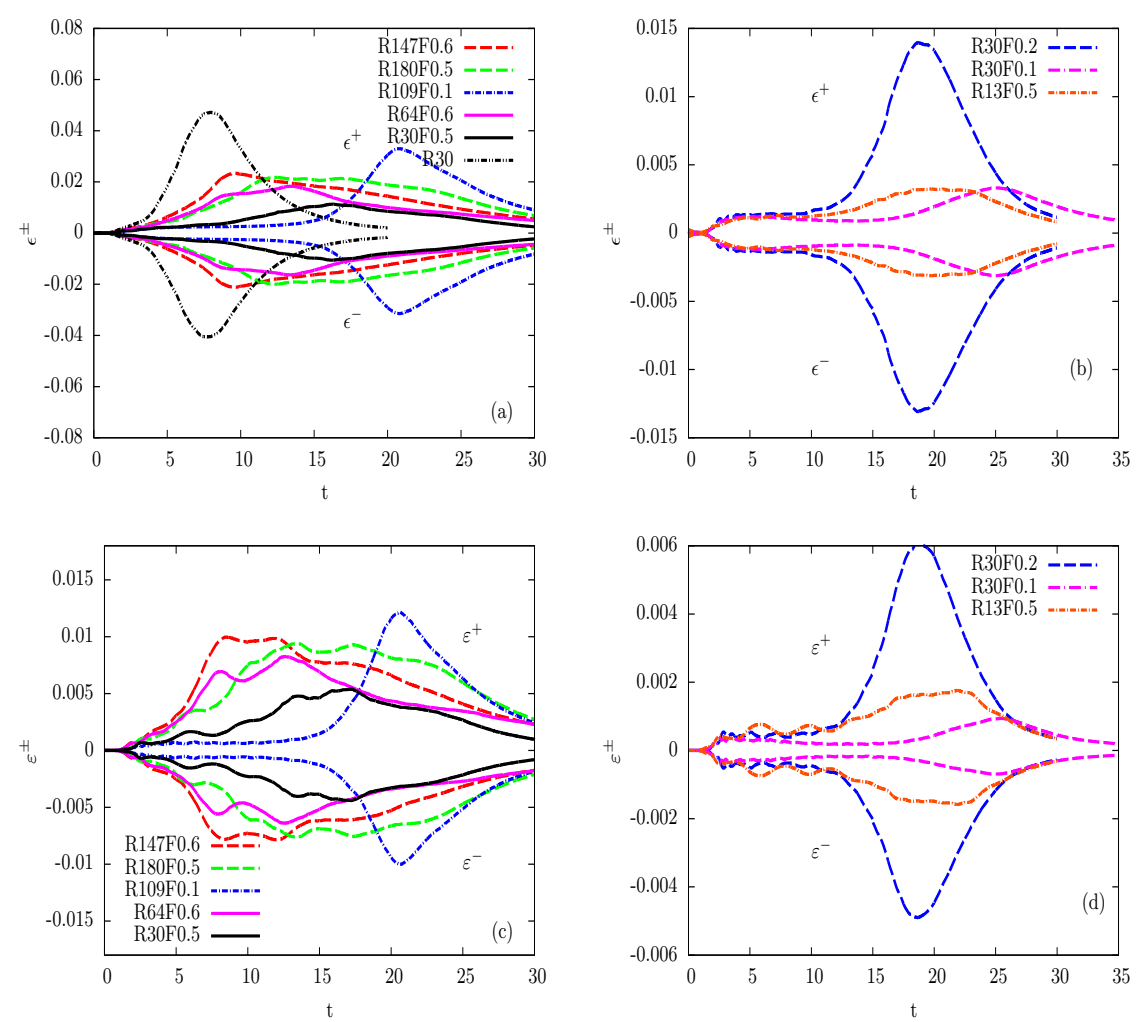

Figure 4: Time series of forward- and backscatter components of the domain-averaged effective SGS dissipation rate at $k_{c}=40$ : (a,b) kinetic and (c,d) potential. The left panels correspond to cases with $R e_{b} \gtrsim 1$ and the right panels show those with $R e_{b} \lesssim 1$. 
and $\epsilon_{S G S}$ and $\varepsilon_{S G S}$ are peaked around the time that the total viscous dissipaor $k_{c} / k_{o}$. In the next section, we investigate the dependence on these ratios in wavenumber space, where more differences become apparent. 

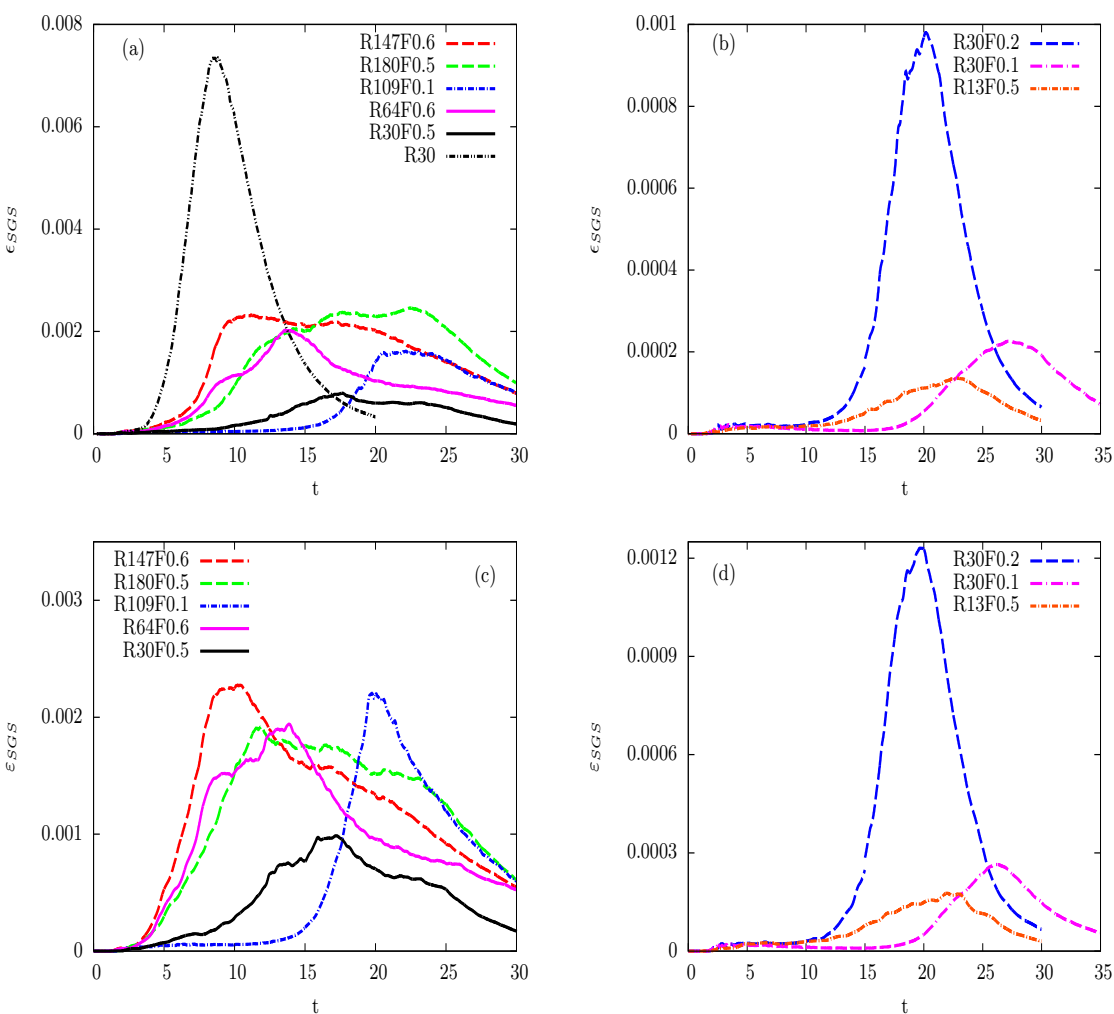

Figure 5: Time series of the domain-averaged effective SGS dissipation rate at $k_{c}=40:(\mathrm{a}, \mathrm{b})$ kinetic and $(\mathrm{c}, \mathrm{d})$ potential. The left panels correspond to cases with $R e_{b} \gtrsim 1$ and the right panels show those with $R e_{b} \lesssim 1$. 

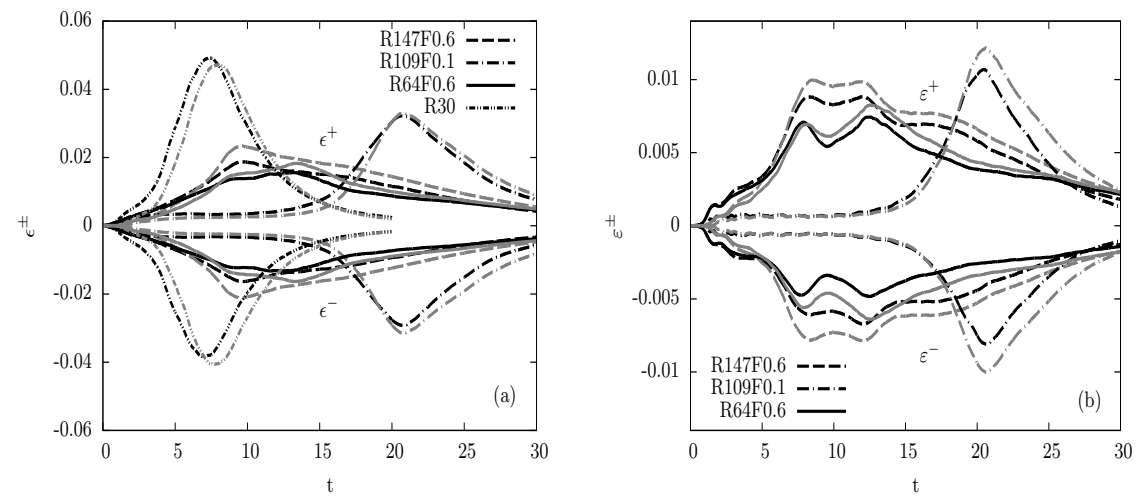

Figure 6: Time series of forward- and backscatter components of the domain-averaged effective SGS dissipation rate for $k_{c}=20$ (black colour) and $k_{c}=40$ (grey colour): (a) kinetic and (b) potential.

\subsection{Energy transfer in wavenumber space}

The averaged effective SGS kinetic and potential energy transfer spectra for $k_{c}=40$ are shown in figure 7 (left panels correspond to cases with $R_{b} \gtrsim 1$ and right panels show $R e_{b} \lesssim 1$ ). According to our definitions in equations (12[13), positive values of $\left\langle T_{k}\left(k \mid k_{c}, t\right)\right\rangle$ and $\left\langle T_{p}\left(k \mid k_{c}, t\right)\right\rangle$ correspond to downscale energy transfer from above to below the filter scale, and negative values denote backscatter. For the unstratified simulation and stratified cases with $R e_{\ell}=18000$ and $F r_{\ell}=0.48, R e_{\ell}=10900$ and $F r_{\ell}=0.16$, and $R e_{\ell}=6400$, 14700 and $F r_{\ell}=0.64$, which are cases with the largest $R e_{b}$, no backscatter is observed at any wavenumber. However, increased stratification along with decreased Reynolds number results in the upscale transfer of kinetic energy over intermediate scales $0.1 \lesssim k / k_{c} \lesssim 0.7$ (figures $7 \mathrm{~b}, \mathrm{~b}$ ). These trends suggest that by reducing $R e_{b}$ to be around 1 or smaller (i.e. reducing the spectral gap between buoyancy and Ozmidov wavenumbers to the viscous dissipation wavenumber $k_{d}$ ), backscatter emerges in stratified turbulence over an intermediate range of wavenumbers (recall, however, that the net SGS transfer, i.e. the sum of these transfer spectra, is still downscale; see figure 5). All simulations with $R e_{b}$ similar to or less than 1 (i.e. $R e_{b} \leq 1.45$ ) exhibit backscatter emerges 

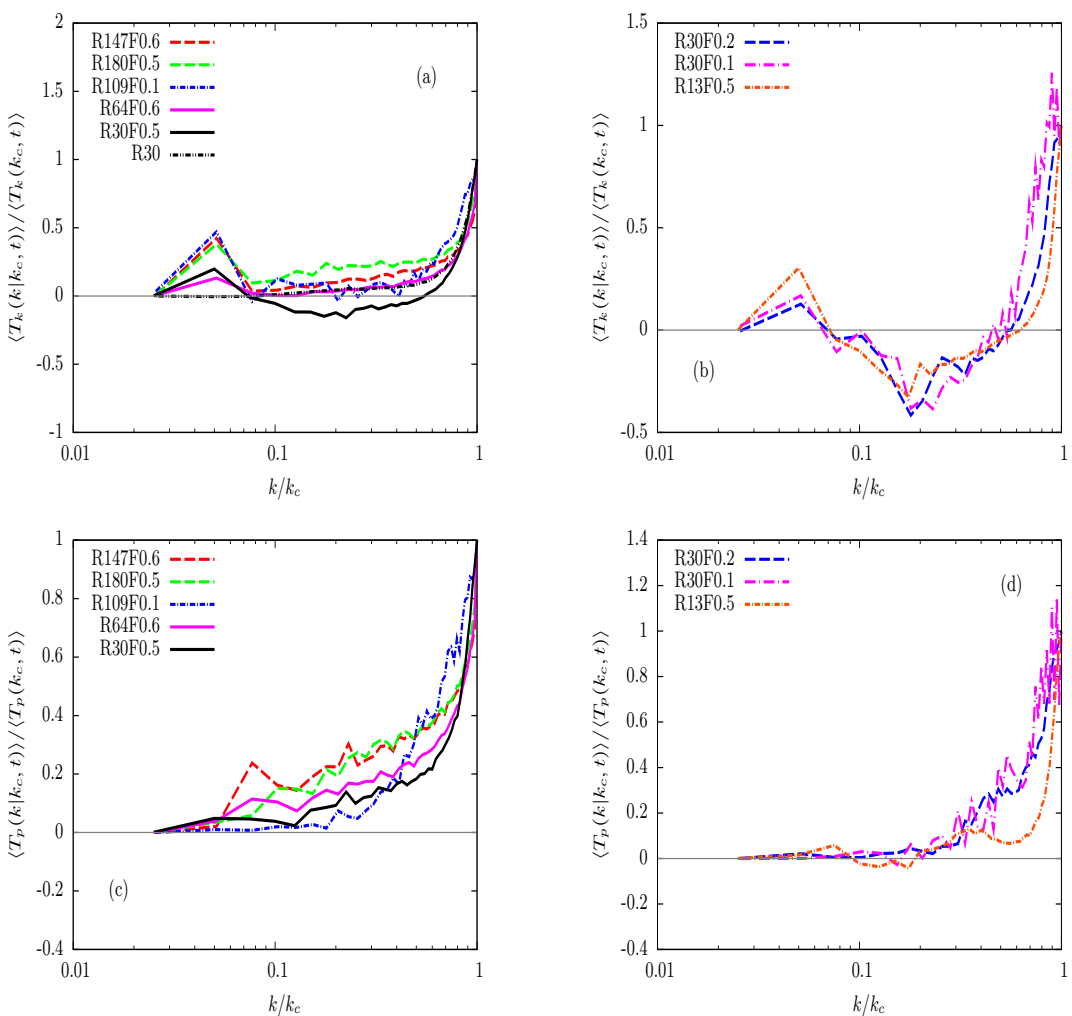

Figure 7: Averaged effective SGS $(a, b)$ kinetic and $(c, d)$ potential energy transfer spectra for $k_{c}=40$. The left panels correspond to cases with $\operatorname{Re}_{b} \gtrsim 1$ and the right panels show those with $R e_{b} \lesssim 1$. The solid grey line indicates zero.

over intermediate wavenumbers. For the case with $\operatorname{Re}_{\ell}=10900$ and $F r_{\ell}=0.16$, backscatter occurs at a few discrete wavenumbers; for all other cases, it occurs for a broad range of intermediate wavenumbers. Spectral backscatter is not seen in the effective SGS potential energy transfer spectra for any parameter values (figures 75, d).

The averaged horizontal and vertical wavenumber effective SGS kinetic and potential energy transfer spectra for the cases with $\operatorname{Re}_{\ell}=3030$ and $F r_{\ell}=0.24$, and $R e_{\ell}=18000$ and $F r_{\ell}=0.48$ are shown in figure 8. It is clear that the case with $R e_{b} \approx 14$ does not show any spectral backscatter in total, horizontal, or vertical wavenumbers (figure 8k, d). However, the case with $R e_{\ell}=3030$ and 
$F r_{\ell}=0.24\left(R e_{b} \approx 1\right)$ shows backscatter at large horizontal and small vertical scales (figure 8a). The sign of the SGS transfer into $k_{h}=0$ is the same as the sign of the transfer into small but non-zero $k_{h}$ (not shown). To further examine this backscatter, and in particular its dependence on Reynolds number, we have performed a series of simulations in which the initial Reynolds number is systematically increased at fixed initial Froude number, thereby increasing the buoyancy Reynolds number. In particular, we consider cases with $F r_{\ell} \approx 0.1$ and $R e_{\ell}=3030,10900$, and $F r_{\ell}=0.48$ and $R e_{\ell}=3030,18000$. Interestingly, increased $R e_{\ell}$ (and hence $R e_{b}$ ) leads to decreasing, and finally an elimination, of the backscatter (see dot-dashed cyan and blue lines, and black solid and dashed green lines in figures $7 \mathrm{a}, \mathrm{b})$. Increased $R e_{\ell}$ leads to a decrease in the Kolmogorov scale $L_{d}$ and an increase in the ratio $\Delta_{\text {test }} / L_{d}$ (see below).

By contrast, the horizontal and vertical effective SGS potential energy transfer spectra do not show backscatter (figure 8 $\mathrm{b}$ ). The lack of backscatter in potential energy could be due to the large-scale flow induced by the initial TG vortices, which have no potential energy [similar results were found in e.g. 21]. Also, it is worthwhile mentioning that the existence of spectral backscatter is not an artifact of the domain size. We have run a new simulation with $R e_{\ell}=3030$ and $F r_{\ell}=0.24$, and $L=4 \pi$, while $\Delta$ is approximately fixed, to evaluate the effects of domain size on the spectral backscatter. The resulting transfer spectrum is similar to that shown in figure 8 a (plots are not shown).

\subsubsection{Effects of changing the test cutoff $k_{c}$}

By decreasing the location of the test cutoff wavenumber $k_{c}$ from 40 to 20 , spectral SGS kinetic and potential energy transfers change dramatically (figures 9). The magnitudes of effective SGS energy transfers are increased using smaller $k_{c}$. In addition, the spectral kinetic energy transfer show less backscatter than was observed with $k_{c}=40$. Indeed, in some cases that showed backscatter with $k_{c}=40$, the backscatter is completely eliminated with $k_{c}=20$. As a result, by moving the test cutoff $k_{c}$ towards small wavenumbers, the upscale energy transfer is reduced or eliminated. For example, the stratified case with $R e_{\ell}=$ 

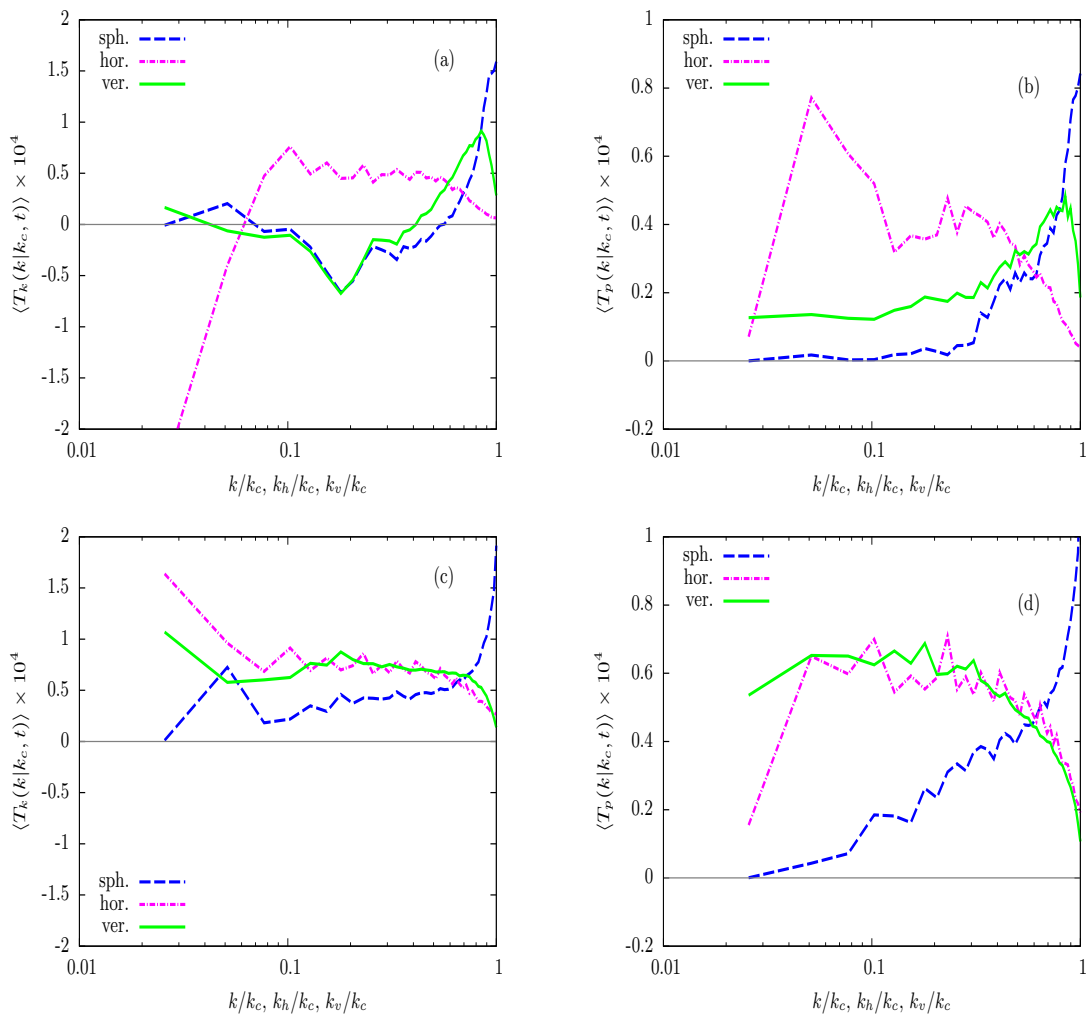

Figure 8: Averaged horizontal and vertical wavenumber effective SGS (a,c) kinetic and (b,d) potential energy transfer spectra for cases with (a,b) $R e_{\ell}=3030$ and $F r_{\ell}=0.24$, and (c,d) $R e_{\ell}=18000$ and $F r_{\ell}=0.48$, when $k_{c}=40$. Spectra are shown as functions of total, horizontal and vertical wavenumbers. The $k$ spectra (blue dashed lines in figure 8 ) show those corresponding curves that were shown in figure 7 

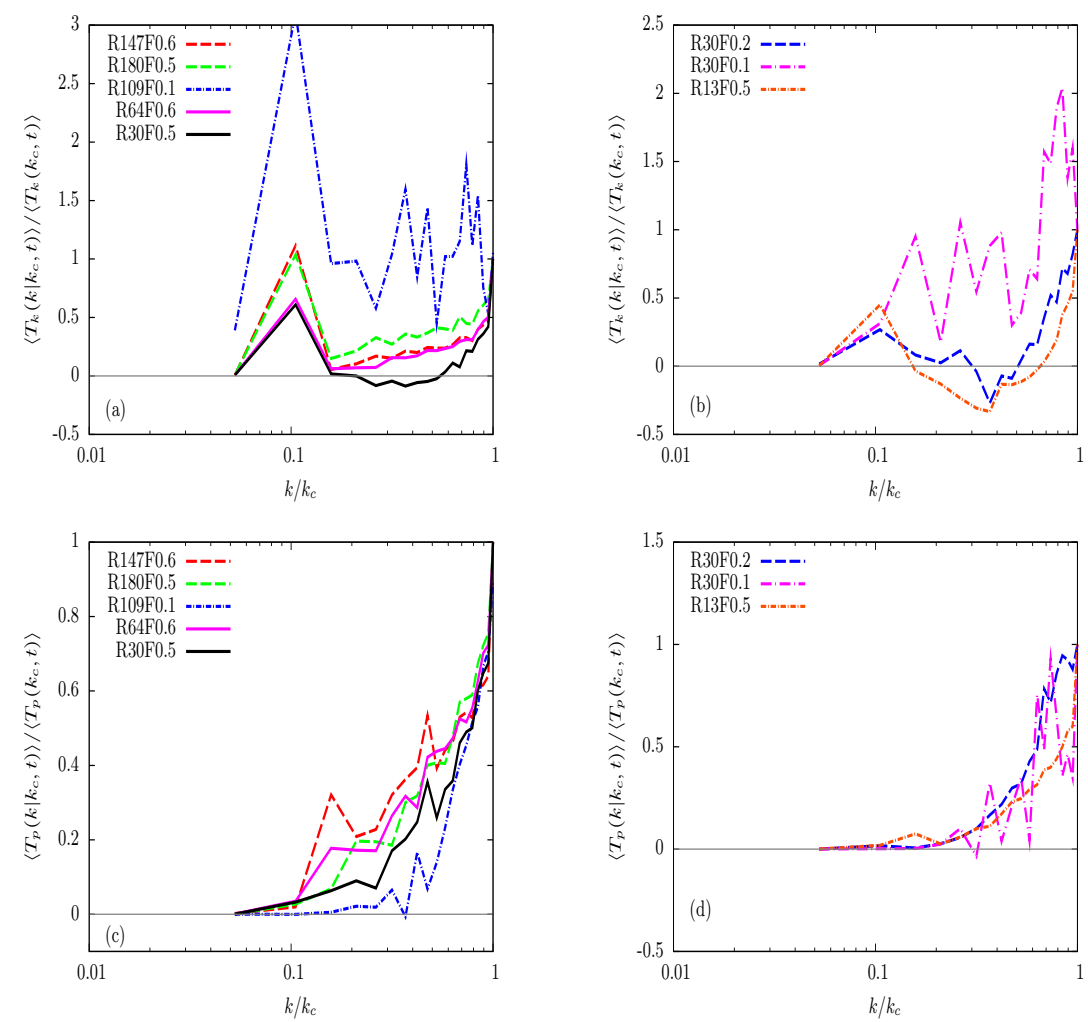

Figure 9: Averaged effective SGS (a,b) kinetic and (c,d) potential energy transfer spectra for $k_{c}=20$. The left panels correspond to cases with $R e_{b} \gtrsim 1$ and the right panels show those with $R e_{b} \lesssim 1$. The solid grey line indicates zero.

3030 and $F r_{\ell}=0.12$ shows no backscatter when $k_{c}=20$ (figure 9p), in contrast with the corresponding results when $k_{c}=40$ (figure $7 \mathrm{~b}$ ). In fact, in almost all of the simulations that showed spectral backscatter with $k_{c}=40$ in figure 7 backscatter is eliminated or occurs over a narrow range of wavenumbers when $k_{c}=20$ as seen in figure 9. These trends suggest that the upscale energy transfer happens from sub-test-filter scales, which are affected by viscous layering for small $R e_{b}$, towards the smaller test-resolved scales (see figures 7 and 9 ).

To investigate the dependence of backscatter on $k_{c}$, we consider the relationship between $k_{c}$ and the viscous scales in stratified turbulence. The viscous scale is traditionally measured by the Kolmogorov scales $L_{d}$; additionally, in 
stratified turbulence at low $R e_{b} \lesssim 1$, stratified turbulence has a viscously layered structure, with vertical layer thickness of $L_{v i s c}=l_{h} / \sqrt{R e}$, at which viscous effects are important [e.g. 17]. In our simulations with $F r_{\ell} \approx 0.1, L_{v i s c} \approx 0.04$ and 0.08 when $R e_{\ell}=10900$ and 3030, respectively. As a result, the test filter scales are clearly affected by viscosity at smaller $R e$, less so at larger $R e$. If we compare $L_{v i s c}$ with the test-filter width $\Delta_{\text {test }}=\pi / k_{c}$, which is around 0.08 for $k_{c}=40$, it is clear that with increasing $R e, L_{v i s c}$ decreases in comparison with $\Delta_{\text {test }}$, which could account for the elimination of backscatter.

In a similar examination at higher $R e_{b}$, we calculate the averaged effective SGS kinetic energy transfer spectra for the high resolution case with $F r_{\ell}=0.64$ when $k_{c}$ moves from 20 to 160 (figure 10). It is clear that by increasing the value of $k_{c}$ from 20 towards the viscous dissipation range (i.e. 160, see the arrows in figure 3a), negative kinetic energy transfer spectra emerges in the intermediate 325 range (figure 10). Consistent with our results in figures 7 and 9 , if we move $\Delta_{\text {test }}$ from $\gg L_{d}$ to $\lesssim L_{d}$, backscatter occurs even at large $R e_{b}$.

Figure 11 summarizes the presence or not of spectral backscatter by systematical plots of the ratios of the test filter scale to the key scales of stratified turbulence: the buoyancy, Ozmidov, viscous and Kolmogorov scales, versus the buoyancy Reynolds number $R e_{b}$ for all simulations when $k_{c}$ varies from 20 to 160. Symbols indicate whether spectral backscatter is observed or not. It is clear from the above that at fixed $R e_{b}$, increased $\Delta_{\text {test }}$ leads to a decrease in spectral backscatter and finally elimination of the spectral upscale transfer (see figures (7) 9). At fixed $k_{c}$ and $F r_{h}$, the spectral backscatter decreases and finally disappears with increased $R e$ (figures 7 , 9). Also, at fixed $R e_{b}$, if we increase $k_{c}$ towards the dissipation range, the spectral energy transfer plateau decreases and even become negative when $k_{c}$ is very close to $k_{d}$ (figures 10 and 112). Figure 11 shows that, at least for $R e_{b} \gtrsim 10$, there is a clear threshold separating cases with and without spectral backscatter when the ratio $\Delta_{t e s t} / L_{d}$ 340 is considered. For $R e_{b} \gtrsim 10$, backscatter occurs when $\Delta_{\text {test }} / L_{d}<1.2$ and is eliminated when this ratio is $>1.2$ (figure 117). In this regime, backscatter depends on both $\operatorname{Re}_{\ell}$ and $F r_{\ell}$ insofar as they determine $R e_{b}$. Beyond that, it 


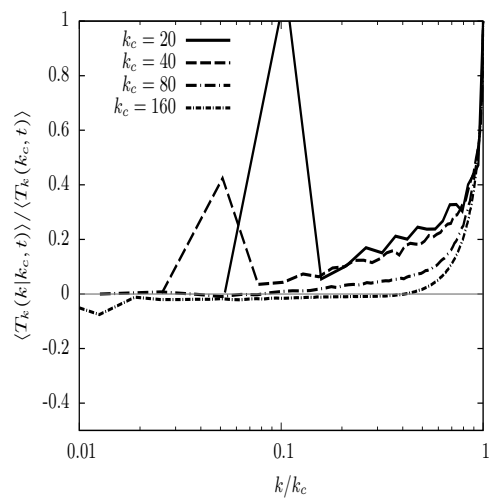

Figure 10: Averaged effective SGS kinetic energy transfer spectra for the high resolution case with $R e_{\ell}=14700$ and $F r_{\ell}=0.64$ when different test cutoff filters $k_{c}$ are applied. The solid grey line indicates zero.

is mainly $R e_{\ell}$ that matters, as it determines $L_{d}$. The behaviour for $R e_{b} \lesssim 1$ is more complicated, but the trend discussed above - backscatter appears for sufficiently small $\Delta_{t e s t}$ - is clear. The lack of a clear threshold on $\Delta_{t e s t} / L_{d}$ at small $R e_{b}$ may be due to the fact that these simulations are on the edge of the stratified turbulence regime.

We speculate that there may be a similar relation between the viscous scale and test-filter width in DNS of Remmler \& Hickel [21], who have also reported spectral backscatter in DNS of stratified turbulence. Using $l_{h}=2 \pi$ in their strongly stratified case where $R e_{b} \approx 2$, we can get $L_{d} \approx 0.03$, which is very close to the test-filter width in their simulation. Hence, we speculate that if their test cutoff filter $k_{c}$ was decreased, their spectral backscatter would also be decreased and finally eliminated. As a result, the upscale energy transfer in wavenumber space could be due to viscous effects rather than a turbulent mechanism. These findings suggest that the upscale energy transfer reported by Remmler \& Hickel 21] may be due to effects of the low Reynolds number and filtering the viscous scale.

When $R e_{b} \gtrsim 1$, the SGS energy transfer spectra display a plateau at intermediate wavenumbers along with a cusp around $k_{c}$ when $k_{c} \gtrsim k_{b}$, consistent 

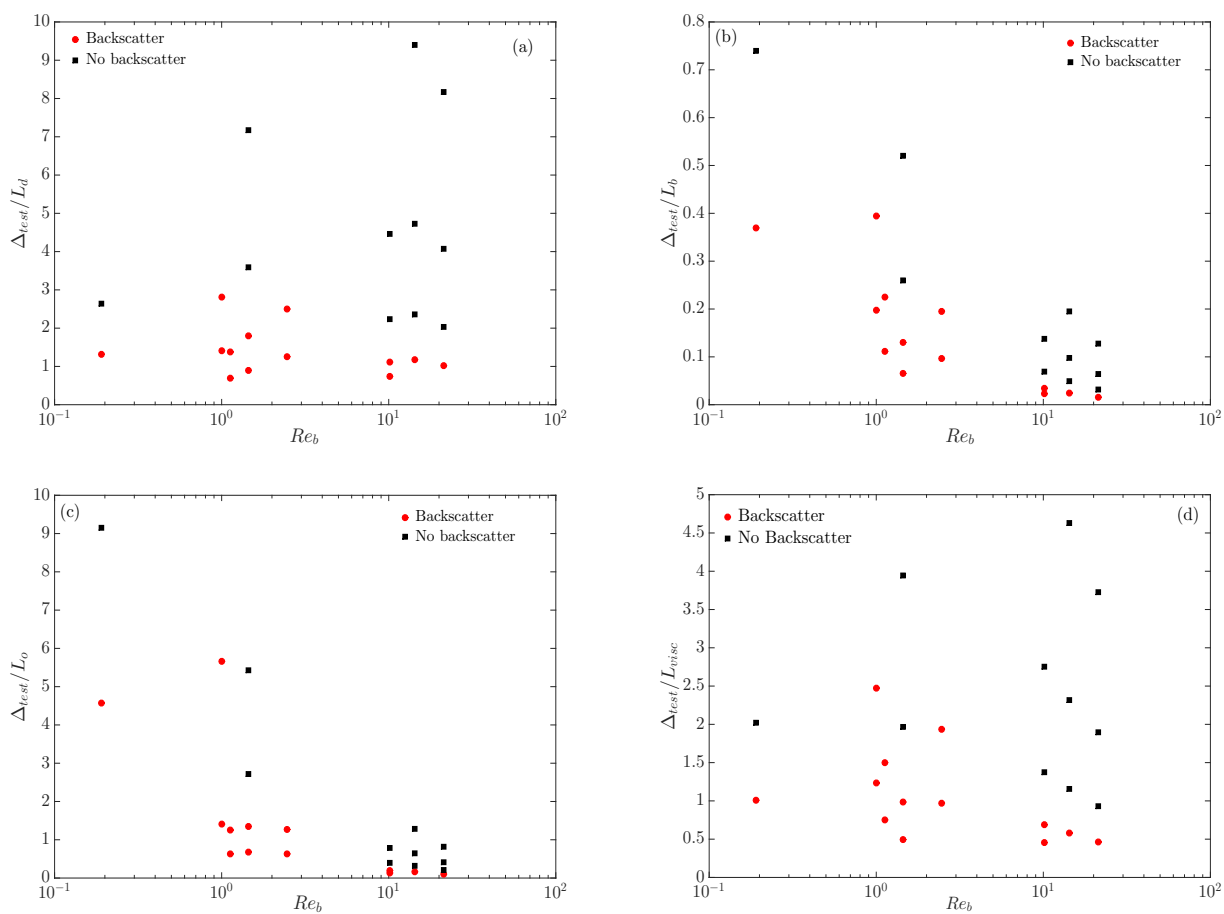

Figure 11: The ratios (a) $\Delta_{\text {test }} / L_{d}$, (b) $\Delta_{\text {test }} / L_{b}$, (c) $\Delta_{\text {test }} / L_{o}$ and (d) $\Delta_{\text {test }} / L_{v i s c}$, versus $R e_{b}$ where the red circles denote observation of spectral backscatter and the black squares show those ratios in which the spectral upscale energy transfer is eliminated; $L_{d}, L_{b}, L_{o}, L_{v i s c}$ and $R e_{b}$ are from the time of maximum $\epsilon$. 
with the classical picture of the Kraichnan 2] eddy viscosity and local energy transfer (figure 77). However, when $k_{c}<k_{b}$, the plateau overcomes the cusp and so the non-local SGS energy transfer becomes dominant (figure 9). In particular, for the cases with $R e_{\ell}=10900,3030$ and $F r_{\ell}=0.1$, the transfers are dominated by a cusp around $k_{c}$ when $k_{c}=40$ (figure $7 \mathrm{~h}, \mathrm{~b}$ ) but are dominated by non-local energy transfer at intermediate wavenumber when $k_{c}=20$ (figures 9a,b), where for both cases $20 \lesssim k_{b}<40$. These trends suggest that there is a non-local kinetic energy transfer from scales larger than $L_{b}$ to small scales. This transfer is consistent with the evolution of the TG vortices, which are initially dominated by $k \sim 1$, into a layerwise flow with $k \sim k_{b}[19,31]$. This nonlocality in SGS energy transfer spectra might underscore the importance of resolving the buoyancy scale in LES of stratified turbulence that has been suggested in the literature [10, 22].

\subsubsection{The effective turbulent Prandtl number}

Figure[12] shows the averaged effective turbulent Prandtl number $\left\langle P r_{t}\left(k \mid k_{c}, t\right)\right\rangle$ spectra at different test cutoffs $k_{c}$ (left panels show results for $R e_{b} \gtrsim 1$ and right panels show those for $R e_{b} \lesssim 1$ ). The cases with $R e_{\ell}=14700,6400$ and $F r_{\ell}=0.64$, and $R e_{\ell}=18000$ and $F r_{\ell}=0.48$ show positive values that are between 0 and 1 , for both test cutoff $k_{c}=20$ and 40 (solid purple, dashed red and green lines in figures $12 \mathrm{a}, \mathrm{c})$. However, all other cases show negative $\left\langle P r_{t}\left(k \mid k_{c}, t\right)\right\rangle$ spectra over the intermediate range $0.1 k_{c} \lesssim k \lesssim 0.6 k_{c}$. By moving $k_{c}$ from 40 to 20 , negative $\left\langle\operatorname{Pr}_{t}\left(k \mid k_{c}, t\right)\right\rangle$ values are decreased for cases with $R e_{\ell}=3030$ and $F r_{\ell}=0.24,0.12$ (figure 12b,d). As shown in above, the negative sign of the effective turbulent Prandtl numbers could be due to filtering the viscous scale (see figures 7, 9 and 10). It is interesting that for all cases, the averaged effective turbulent Prandtl number is positive and fairly close to 1 for $k$ around $k_{c}$. This trend suggests that the assumption of $P r_{t} \approx 1$ is reasonable for the local energy transfer at all regimes of $R e_{b}$ [as is assumed in e.g. 10, 22, 32]. However, by decreasing $R e_{b}$ to be of order $\sim 1$ or $\ll 1$, this assumption might not be valid for the nonlocal energy transfer when $\Delta_{t e s t} \lesssim L_{v i s c}$. 

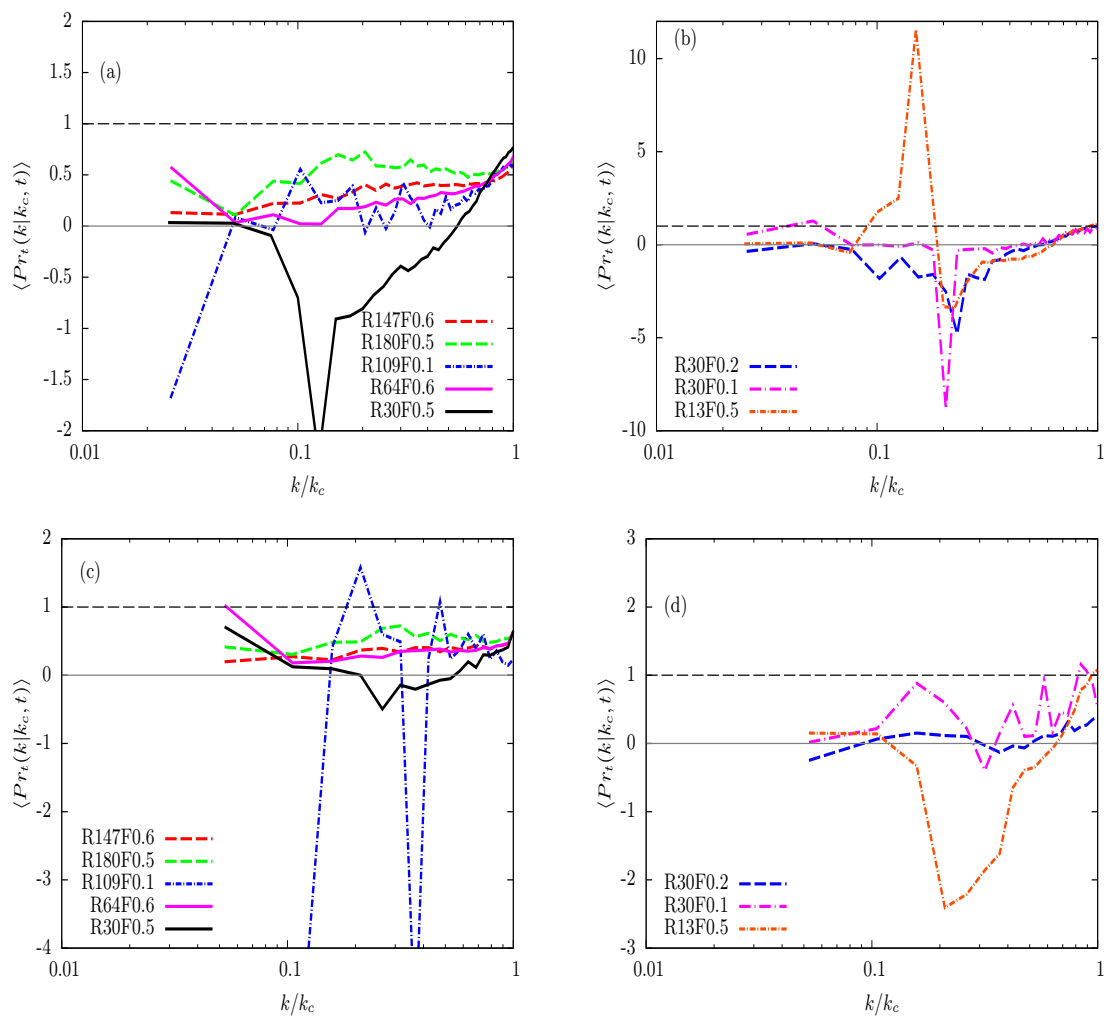

Figure 12: Averaged effective turbulent Prandtl number spectra for $(\mathrm{a}, \mathrm{b}) k_{c}=40$ and $(\mathrm{c}, \mathrm{d})$ $k_{c}=20$. The left panels correspond to cases with $R e_{b} \gtrsim 1$ and the right panels show those with $R e_{b} \lesssim 1$. The solid grey line and dashed black line indicate values of zero and 1 , respectively. 


\section{Conclusion}

The dynamics of kinetic and potential energy transfer around a test cutoff filter in both physical and wavenumber space for decaying stratified turbulence is studied in this paper. The effective domain-averaged SGS energy transfer

$\epsilon_{S G S}$ is a small net energy transfer from large to small scales that results from the combination of two large energy transfer mechanisms: downscale $\epsilon^{+}$and upscale $\epsilon^{-}$[in line with results for unstratified simulations, e.g. 4]. Increased buoyancy Reynolds number $R e_{b}$ leads to an increase in the upscale and downscale kinetic and potential SGS energy transfer, and hence increasing effective SGS energy transfer in physical space. As a result, the domain-averaged upscale energy transfer might not be just a random and stochastic process that is independent of the flow dynamics, as is considered for modelling backscatter in the literature [e.g. 33, 34, 35, 36]. It is also shown that $\epsilon^{ \pm}$and $\varepsilon^{ \pm}$are not very sensitive to the location of the test cutoff $k_{c}$. Our results show that the domainaveraged backscatter depends on the parameters of stratified turbulence such as the Froude and buoyancy Reynolds numbers. A similar idea is suggested by O'Brien et al. [27], in which backscatter in reacting turbulence depends on the dynamics of the flow such as the SGS Mach number and high-speed compressibility.

In wavenumber space, the effective kinetic and potential energy transfer spectra are positive (i.e. forward scatter) when $R e_{b} \sim O(10)$. However, for $R e_{b} \sim O(1)$ or smaller, the kinetic energy transfer spectra show negative values (i.e. backscatter) when the Reynolds number is small. This behaviour occurs when the test filter scale falls below the scale of the viscous layers that are present at small $R e_{b}$, or when $\Delta_{\text {test }} \sim L_{d}$ even at $R e_{b} \gtrsim 1$. These trends suggest that the effective kinetic energy transfer spectra are contaminated by viscous effects in the small Reynolds number regime, in which backscatter are seen in the effective kinetic energy transfer spectra. In addition, our results show a non-local energy transfer from scales larger than $L_{b}$ to small scales. Recently, an a posteriori study on LES of stratified turbulence has shown that current 
eddy-viscosity SGS models (i.e. the Smagorinsky, dynamic Smagorinsky and Kraichnan models) require resolution of $L_{b}$ to capture the fundamental features of stratified turbulence [10, 22]. DNS results of this paper suggest that the requirement of resolving $L_{b}$ in LES of stratified turbulence is related to the need of resolving the non-local energy transfer around $L_{b}$, since current SGS models are developed based on the local SGS dissipation, i.e. removing energy mainly at smallest resolved scale $\Delta$. The effective turbulent Prandtl number spectra suggest that the assumption of $P r_{t}=1$ is reasonable for the local energy transfer. For the non-local energy transfer, however, the effective $\left\langle P r_{t}\right\rangle$ is negative when $\operatorname{Re}_{b} \sim O(1)$ or smaller at $\Delta_{\text {test }} \sim L_{d}$.

Performing DNS of stratified turbulence for large and small $R e_{b}$, when the Reynolds number is very large is a potential direction for future work, so that the dynamics of energy transfer around the buoyancy and Ozmidov scales could be studied in detail, and away from the effects of molecular viscosity where $L_{d}$ 435 is very small. Also, applying different types of test filters, in particular those are common in physical space, such as the top-hat or Gaussian filters, is desirable to study the sensitivity of backscatter to the type of test filter. Moreover, it would be interesting to study spectral backscatter in simulations with largescale potential energy forcing/initial conditions, e.g. large-scale gravity waves.

${ }_{440}$ In addition, an a priori evaluation of current SGS models in DNS of stratified turbulence will be another research avenue towards developing a new SGS model that does not require resolving $L_{b}$ in LES of stratified turbulence.

\section{Acknowledgement}

This work benefited from comments by J. A. Domaradzki, K. G. Lamb and M. Stastna and two anonymous reviewers. Computations were performed on the gpc supercomputer at the SciNet HPC Consortium. SciNet is funded by: the Canada Foundation for Innovation under the auspices of Compute Canada; the Government of Ontario; Ontario Research Fund-Research Excellence; and the University of Toronto. Also, This work was made possible by the facilities 
of the Shared Hierarchical Academic Research Computing Network (SHARCNET: www.sharcnet.ca) and Compute/Calcul Canada. In addition, computing resources from the Mathematical Faculty Computing Facility of the University of Waterloo are gratefully appreciated. Financial support from the Natural Sciences and Engineering Research Council of Canada is gratefully acknowledged.

[1] J. Smagorinsky, General circulation experiments with the primitive equations. I. The basic experiment Mon. Weather Rev. 91(3), 99-164 (1963).

[2] R. H. Kraichnan, Eddy viscosity in two and three dimensions. J. Atmos. Sci. 33, 1521-1536 (1976).

[3] L. F. Richardson, Weather prediction by numerical process. Cambridge University Press, Cambridge (1922).

[4] U. Piomelli, W. H. Cabot, P. Moin, and S. Lee, Subgrid-scale backscatter in turbulent and transitional flows. Phys. Fluids A 3(7), 1766-1771 (1991).

[5] J. A. Domaradzki, W. Liu, and M. E. Brachet, An analysis of subgrid-scale interactions in numerically simulated isotropic turbulence. Phys. Fluids A 5, 1747-1759 (1993).

[6] M. Germano, U. Piomelli, P. Moin, and W. H. Cabot, A dynamic subgrid-scale eddy viscosity model. Phys. Fluids A 3(7), 1760-1765 (1991).

[7] S. Ghosal, T. S. Lund, P. Moin, and K. Akselvoll, A dynamic localization model for large-eddy simulation of turbulent flows. J. Fluid Mech. 286, 229-255 (1995).

[8] C. Meneveau and J. Katz, Scale-invariance and turbulence models for large-eddy simulation. Ann. Rev. Fluid Mech. 32, 1-32 (2000). 
[9] S. B. Pope, Turbulent Flows. Cambridge University Press, Cambridge (2000).

[10] S. Khani and M. L. Waite, Large eddy simulations of stratified turbulence: the dynamic Smagorinsky model. J. Fluid Mech. 773, 327-344 (2015).

[11] S. Remmler and S. Hickel, Direct and large eddy simulation of stratified turbulence. Int. J. Heat Fluid Flow 35, 13-24 (2012).

[12] F. Wan and F. Porté-Agel, Large-eddy simulation of stably-stratified flow over a steep hill. Boundary-Layer Meteorol 138, 367-384 (2011).

[13] J. J. Riley and M.-P. Lelong, Fluid motions in the presence of strong stable stratification. Annu. Rev. Fluid Mech. 32, 613-657 (2000).

[14] S. Almalkie and S. M. de Bruyn Kops, Kinetic energy dynamics in forced, homogeneous, and axisymmetric stably stratified turbulence. J. Turbul. 13 1-32 (2012).

[15] P. Bartello and S. M. Tobias, Sensitivity of stratified turbulence to the buoyancy Reynolds number. J. Fluid Mech. 725, 1-22 (2013).

[16] P. Billant and J.-M. Chomaz, Self-similarity of strongly stratified inviscid flows. Phys. Fluids 13(6), 1645-1651 (2001).

[17] G. Brethouwer, P. Billant, E. Lindborg, and J.-M. Chomaz, Scaling analysis and simulation of strongly stratified turbulent flows. J. Fluid Mech. 585, 343-368 (2007).

[18] E. Lindborg, The energy cascade in strongly stratified fluid. J. Fluid Mech. 550, 207-242 (2006).

[19] M. L. Waite and P. Bartello, Stratified turbulence dominated by vortical motion. J. Fluid Mech. 517, 281-303 (2004). 
[20] S. Khani and M. L. Waite, Effective eddy viscosity in stratified turbulence. J. of Turbl. 14(7), 49-70 (2013).

[21] S. Remmler and S. Hickel, Spectral eddy viscosity of stratified turbulence. J. Fluid Mech. 755, R6, doi:10.1017/jfm.2014.423 (2014).

[22] S. Khani S. and M. L. Waite, Buoyancy scale effects in large-eddy simulations of stratified turbulence. J. Fluid Mech. 754, 75-97 (2014).

[23] J. J. Riley and S. M. de Bruyn Kops, Dynamics of turbulence strongly influenced by buoyancy. Phys. Fluids 15, 2047-2059 (2003).

[24] M. L. Waite, Stratified turbulence at the buoyancy scale. Phys. Fluids A 23, 066602 (2011).

[25] C. Rorai, D. Rosenberg, A. Pouquet, and P. D. Mininni, Helicity dynamics in stratified turbulence in the absence of forcing. Phys. Rev. E 87, 063007 (2013).

[26] C. Rorai, P. D. Mininni, and A. Pouquet, Turbulence comes in bursts in stably stratified flows. Phys. Rev. E 89, 043002 (2014).

[27] J. O'Brien, J. Urzay, M. Ihme, P. Moin, and A. Saghafian, Subgridscale backscatter in reacting and inert supersonic hydrogen-air turbulent mixing layers. J. Fluid Mech. 743, 554-584 (2014).

[28] R. Marino, P. D. Mininni, D. L. Rosenberg, and A. Pouquet, Largescale anisotropy in stably stratified rotating flows. Phys. Rev. E 90, 023018 (2014).

[29] R. Marino, A. Pouquet, and D. Rosenberg, Resolving the paradox of oceanic large-scale balance and small-scale mixing. Phys. Rev. Lett. 114, $114504(2015)$.

[30] M. L. Waite, Direct numerical simulations of laboratory-scale stratified turbulence. Modelling Atmospheric and Oceanic Flows: Insights 
from Laboratory Experiments, (eds. T. von Larcher \& P. Williams), American Geophysical Union, Washington, DC., 159-175 (2014).

[31] P. Billant and J.-M. Chomaz, Experimental evidence for a new instability of a vertical columnar vortex pair in a strongly stratified. J. Fluid Mech. 418, 167-188 (2000).

[32] D. A. Siegel and J. A. Domaradzki, Large-eddy simulation of decaying stably stratified turbulence. J. Phys. Oceanogr. 24, 2353-2386 (1994).

[33] J. R. Chasnov, Simulation of the Kolmogorov inertial subrange using an improved subgrid model. Phys. Fluids A 3(1), 188-200 (1991).

[34] M. Lesieur, Turbulence in Fluids. Kluwer Acad. Publrs. Dordrecht (1990).

[35] L. Marstorp, G. Brethouwer, and A. V. Johansson, A stochastic subgrid model with application to turbulent flow and scalar mixing. Phys. Fluids 19, 035107 (2007).

[36] S. Weinbrecht and P. J. Mason Stochastic backscatter for cloudresolving models. Part I: implementation and testing in a dry convective boundary layer. J. Atmos. Sci. 65, 123-139 (2008). 